\title{
Original
}

\section{Trends in domiciliary dental care including the need for oral appliances and dental technicians in Japan}

\author{
Shingo Kamijo'), Kumiko Sugimoto2), Meiko Oki1), Yumi Tsuchida2), \\ and Tetsuya Suzuki2)
1)Department of Basic Oral Health Engineering, Graduate School of Medical and Dental Sciences, Tokyo Medical and Dental University, Tokyo, Japan
2)Department of Oral Prosthetic Engineering, Graduate School of Medical and Dental Sciences, Tokyo Medical and Dental University, Tokyo, Japan

(Received February 14, 2018; Accepted March 27, 2018)

\begin{abstract}
The need for domiciliary dental care (DDC) for people requiring long-term nursing care is increasing as the super-aged society of Japan grows still older. Dysphagia diagnosis and rehabilitation are becoming more important in DDC; thus, the need for prostheses used for dysphasia rehabilitation is presumed to be increasing. To identify DDC trends in Japan, as well as the need for prostheses and dental technicians for DDC, we sent a selfadministered questionnaire to dentists providing DDC and analyzed responses from 138 dentists (valid response rate, $39.8 \%$ ). The results showed that $37.7 \%$ of respondents reported treating $\geq \mathbf{5 0}$ patients per month. The most frequently performed procedures were removable prosthetic treatment and oral care, followed by dysphagia rehabilitation. Use of palatal augmentation prostheses was experienced by $54.3 \%$ of respondents, and most indicated that the prostheses were effective for improvement of oropharyngeal function. The rates of cooperation with primary care doctors and nursing care professionals were $\mathbf{7 6 . 8 \%}$ and $85.5 \%$, respectively. Only $6.5 \%$ of respondents reported accompanying dental technicians to DDC.
\end{abstract}

Correspondence to Dr. Kumiko Sugimoto, Department of Oral Prosthetic Engineering, Graduate School of Medical and Dental Sciences, Tokyo Medical and Dental University, 1-5-45 Yushima, Bunkyo-ku, Tokyo 113-8549, Japan

Fax: +81-3-5803-0237 E-mail: ksugimoto.bohs@tmd.ac.jp

J-STAGE Advance Publication: October 26, 2018

Color figures can be viewed in the online issue at J-STAGE.

doi.org/10.2334/josnusd.18-0053

DN/JST.JSTAGE/josnusd/18-0053
The present analysis of trends in DDC indicates that oral care and dysphagia rehabilitation have become more frequent and that cooperation with healthcare professionals other than dental technicians has increased in recent DDC.

Keywords: domiciliary dental care; dysphagia rehabilitation; oral appliance; team approach; dental technician.

\section{Introduction}

The aged population of Japan has increased rapidly, reaching 27.3\% in 2016 (http://www8.cao.go.jp/kourei/ whitepaper/w-2017/html/gaiyou/s1_1.html). This trend has greatly increased the number of individuals needing long-term nursing care. Because the capacity of nursing care facilities is insufficient for the population requiring this type of specialized care, many elders live in the community and require doctor visits at home. Similarly, the need for domiciliary dental care (DDC) is believed to have increased recently. However, a few questionnaire studies (Hirata et al., J J Gerodont 27, 276-284, 2012) (1) of DDC provided by members of dental associations in Tokyo reported a relatively low rate of DDC service provision by dentists: $36.6 \%$ in 2006 and $29.2 \%$ in 2011 . These findings suggest that DDC services may not be expanding in metropolitan areas, even though the number of patients was reported to be increasing gradually. Rising rates of aspiration pneumonia have been a public health concern for the elderly population of Japan (http:// www.mhlw.go.jp/toukei/saikin/hw/jinkou/kakutei16/ 
Table 1 Questionnaire on domiciliary dental care (DDC) (questions and response options)

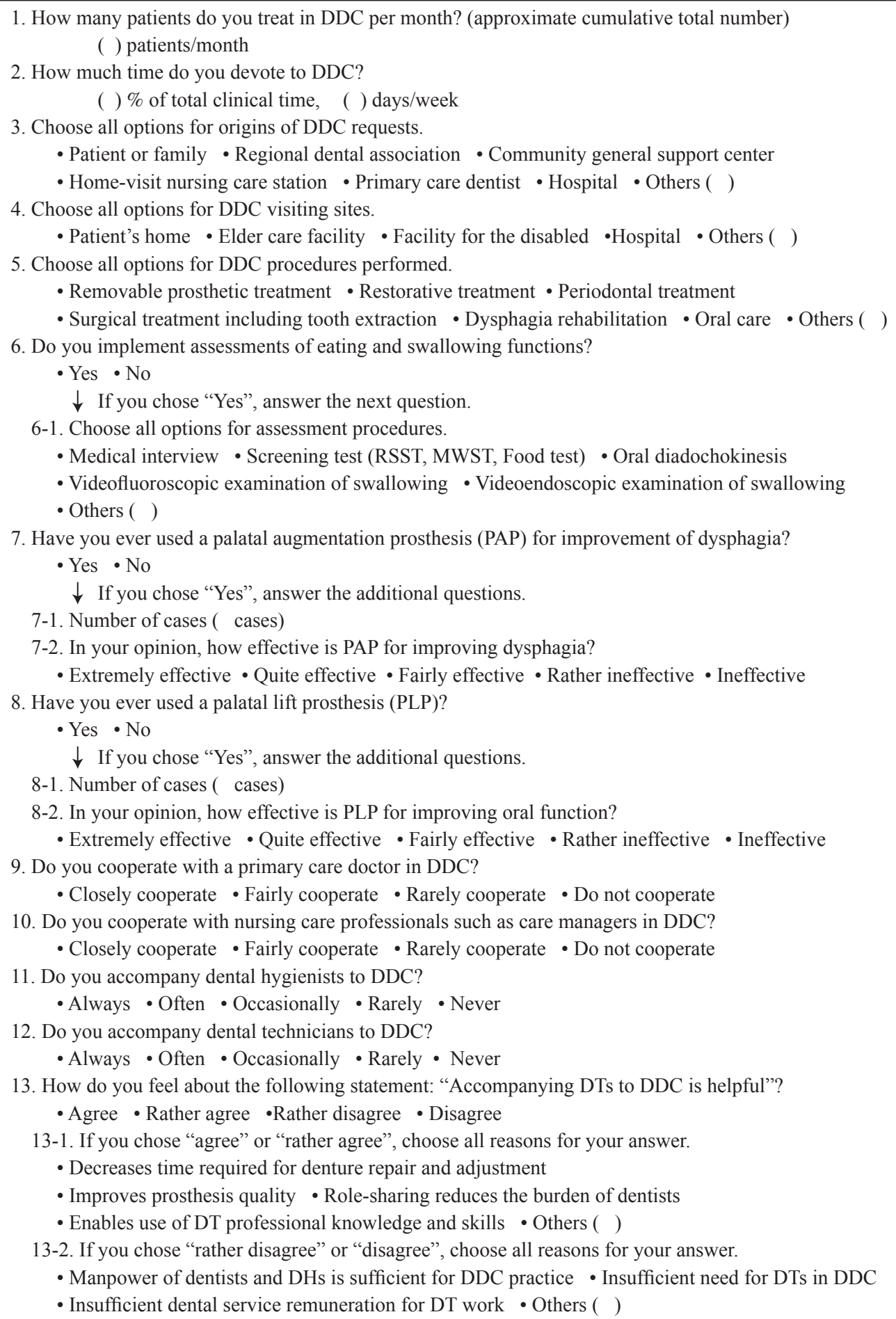

14. How do you feel about the following statement: "Allowing DTs to touch the inside of patients' mouths will improve DDC"?

- Agree • Rather agree • Rather disagree • Disagree

RSST: repetitive saliva swallowing test (Oguchi K et al., Jpn J Rehabil Med 37, 383, 2000), MWST: modified water swallowing test, FT: food test.

d1/00_all.pdf). Therefore, DDC procedures for dysphagia diagnosis and rehabilitation are increasingly important. In this treatment context, there is likely a greater need for oral appliances used for dysphagia rehabilitation, such as palatal augmentation prosthesis (PAP) and palatal lift prosthesis (PLP). However, few studies have examined the need for these appliances in DDC.

Comprehensive community care for people requiring long-term care is expanding in Japan, and interprofessional cooperation and team approaches are essential for 


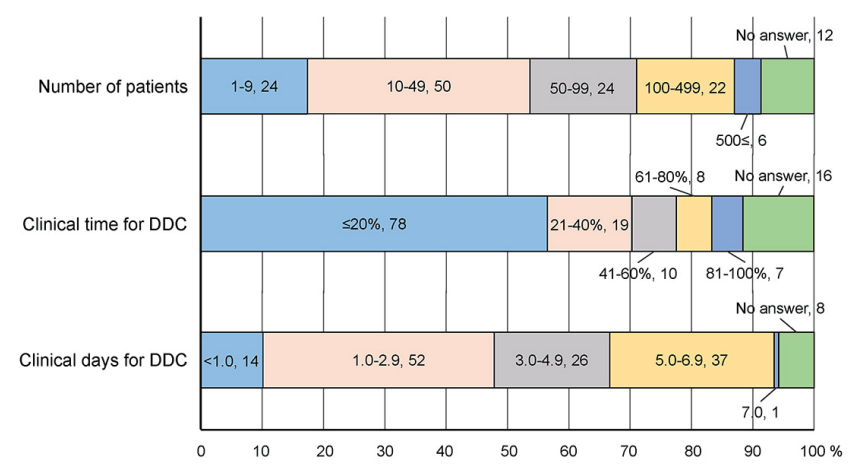

Fig. 1 Status of domiciliary dental care (DDC) practice: number of patients per month, ratio of clinical time spent on DDC, and days per week spent on DDC. The class and number of respondents are indicated in each bar.

such care. In team dental care in DDC, dentists perform dental treatments, and dental hygienists (DHs) provide oral care and assume an important role in managing long-term in-home dental care (2,3). In contrast, dental technicians (DTs) are rarely involved in DDC. However, inclusion of DTs in the DDC team could improve work efficiency and DDC quality. To identify current trends in DDC practice, the need for prostheses in DDC, and the expected role of DTs in DDC, we conducted a selfadministered questionnaire study of dentists providing DDC in Japan.

\section{Materials and Methods}

Self-administered questionnaires were mailed to 347 dentists who were members of the "Home Dental Care Net" and providing DDC in Japan. The respondents were requested to answer the questions and send back the completed questionnaire, only if they understood the questionnaire explanation of the objective and significance of the study and its privacy protections and were willing to cooperate with this study. The survey was conducted from June through July 2016. The questionnaire included items on conditions of DDC practice, the need for oral appliances such as PAP and PLP, cooperation with other healthcare professionals, and the role of DTs in DDC (Table 1).

The collected data were tabulated, and associations between question items were analyzed by using Spearman rank correlation coefficients, the chi-square test, or the Fisher exact test. Multiple logistic analysis using a stepwise method was utilized to identify factors affecting provision of dysphagia rehabilitation. A $P$-value of $<0.05$ was considered to indicate statistical significance. Statistical analyses were performed with SPSS version 23.0 (IBM Corporation, Armonk, NY, USA) for Windows
A. Origin of DDC request (Multiple choice)

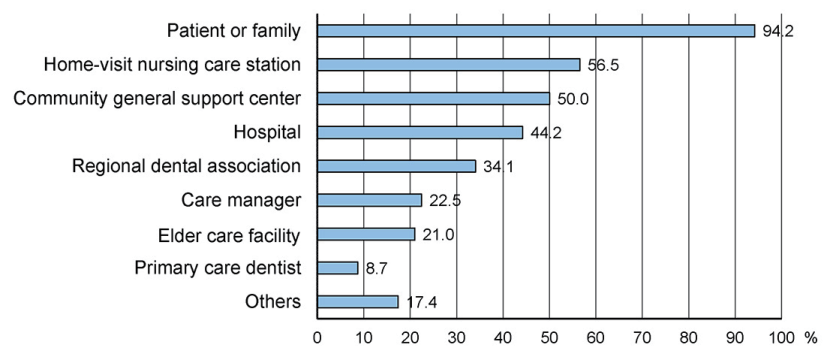

B. Visiting site (Multiple choice)

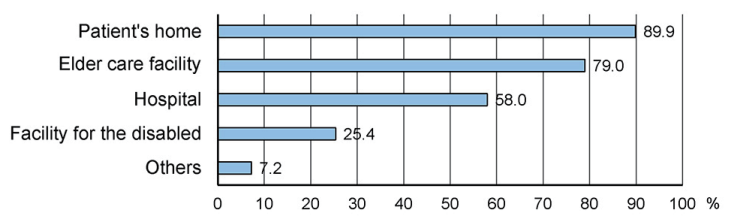

Fig. 2 A: Origins of domiciliary dental care (DDC) requests. "Care manager" and "elder care facility" were extracted from the response "others". B: Types of visiting sites. The percentages of respondents are indicated at the right of each bar.

(Microsoft Corporation, Redmond, WA, USA). This study was conducted with the approval of the Ethics Committee of Faculty of Dentistry, Tokyo Medical and Dental University (approval no. D2016-008).

\section{Respondents}

\section{Results}

Of the 347 dentists who were sent questionnaires, 139 responded to the survey (response rate, 40.1\%), and 138 questionnaires (39.8\%) were deemed valid and analyzed.

\section{Status of DDC}

\section{DDC practice}

Approximately 54\% of respondents were treating fewer than 50 patients per month, while $20.3 \%$ were treating 100 or more (Fig. 1). Regarding time spent on DDC, 56.5\% of respondents answered $<20 \%$ of total clinical time, and $18.1 \%$ answered $>40 \%$. When respondents were asked to describe the time spent on DDC in days per week, $47.8 \%$ of respondents answered $<3$ days and $27.5 \%$ answered 5-7 days. In addition, the number of patients treated per month was highly positively correlated with the ratio of time spent on DDC to total clinical time $(r=0.617, P<$ 0.001 , Spearman rank correlation coefficient) and days per week spent on DDC $(r=0.657, P<0.001)$.

In the analysis of the origin of DDC requests, the patient or family was selected most (94.2\%), followed by home-visit nursing care stations (56.5\%) and community general support centers (50.0\%) (Fig. 2A). The most selected visiting sites were the patient's home (89.9\%) 
Table 2 Associations of number of patients per month with the study variables

\begin{tabular}{|c|c|c|c|c|}
\hline \multirow[b]{2}{*}{ Factor } & & \multicolumn{2}{|c|}{ Number of patients/month } & \multirow{3}{*}{$P$-value* } \\
\hline & & $<35$ & $\geq 35$ & \\
\hline \multirow{2}{*}{\multicolumn{5}{|c|}{ Origin of DDC request }} \\
\hline & & & & \\
\hline \multirow[t]{2}{*}{ Home-visit nursing care station } & Yes & $28(39.4)$ & $43(60.6)$ & \multirow{2}{*}{0.013} \\
\hline & No & $34(61.8)$ & $21(38.2)$ & \\
\hline \multirow[t]{2}{*}{ Community general support center } & Yes & $26(40.0)$ & $39(60.0)$ & \multirow{2}{*}{0.033} \\
\hline & No & $36(59.0)$ & $25(41.0)$ & \\
\hline \multirow[t]{2}{*}{ Primary care dentist } & Yes & $0(0.0)$ & $12(100.0)$ & \multirow{2}{*}{$<0.001$} \\
\hline & No & $62(54.4)$ & $52(45.6)$ & \\
\hline \multicolumn{5}{|l|}{ Visiting site } \\
\hline \multirow[t]{2}{*}{ Elder care facility } & Yes & $43(41.3)$ & $61(58.7)$ & \multirow{2}{*}{$<0.001$} \\
\hline & No & $17(89.5)$ & $2(10.5)$ & \\
\hline \multirow[t]{2}{*}{ Hospital } & Yes & $30(39.5)$ & $46(60.5)$ & \multirow{2}{*}{0.009} \\
\hline & No & $30(63.8)$ & $17(36.2)$ & \\
\hline \multirow[t]{2}{*}{ Facility for the disabled } & Yes & $11(32.4)$ & $23(67.6)$ & \multirow{2}{*}{0.024} \\
\hline & No & $49(55.1)$ & $40(44.9)$ & \\
\hline \multirow[t]{2}{*}{ Cooperation with nursing care professionals } & Yes & $50(45.5)$ & $60(54.5)$ & \multirow{2}{*}{0.027} \\
\hline & No & $12(75.0)$ & $4(25.0)$ & \\
\hline
\end{tabular}

*Chi-square test or Fisher exact test.

DDC: domiciliary dental care.

\section{A. Procedures performed during DDC (Multiple choice)}

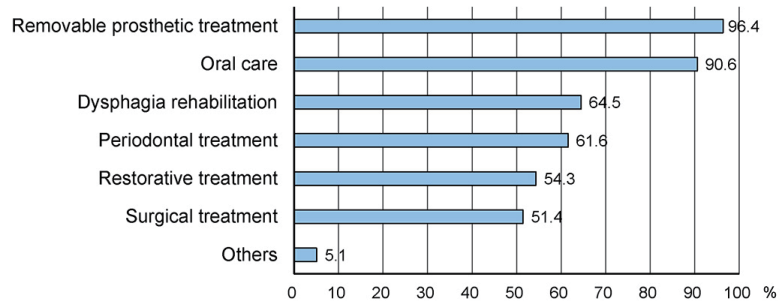

B. Contents of dysphagia assessment (Multiple choice)

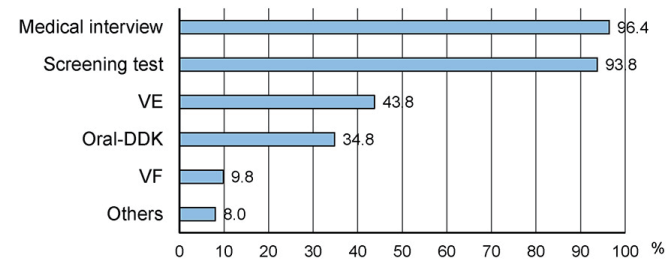

Fig. 3 Procedures performed during domiciliary dental care (DDC) (A), and procedures used for dysphagia assessment by respondents performing such assessments (B). The percentages of respondents are indicated at the right of each bar. VE: video endoscopic examination of swallowing; Oral-DDK: oral diadochokinesis test; VF: videofluoroscopic examination of swallowing.

and elder care facilities (79.0\%) (Fig. 2B).

When respondents were divided into two groups in relation to the median number of patients per month, number of patients per month was significantly associated with several factors, including origin of request, visiting site, and cooperation with other healthcare professionals $(P<0.05$, chi-square test or Fisher exact test) (Table 2).

\section{Content of DDC}

Multiple-choice responses to the question on DDC procedures performed revealed that removable prosthetic treatment and oral care were performed by $96.4 \%$ and $90.6 \%$ of respondents, respectively, followed by dysphagia rehabilitation (provision rate, 64.5\%) (Fig. $3 \mathrm{~A})$.

In addition, eating and swallowing function were assessed by $81.2 \%$ of all respondents, and by virtually all $(96.6 \%)$ of respondents providing dysphagia rehabilitation. Multiple-choice responses on the content of assessment among respondents practicing assessment revealed that a medical interview was performed by $96.4 \%$ of respondents and that a screening test including a repetitive saliva swallowing test $(4,5)$, modified water swallowing test (6-8), and food test (6) was performed by 93.8\% of respondents (Fig. 3B). Furthermore, video endoscopic examination of swallowing (VE) was performed by $43.8 \%$ of respondents, and an oral-diadochokinesis (oral-DDK) testing was performed by $34.8 \%$.

Factors that were significantly associated with provision of dysphagia rehabilitation are shown in Table $3(P<$ 0.05 , chi-square test). As compared with respondents not providing dysphagia rehabilitation services, respondents who did provide such services were treating a larger number of patients, receiving more DDC requests from 
Table 3 Associations of provision of dysphagia rehabilitation with the study variables

\begin{tabular}{|c|c|c|c|c|}
\hline \multirow[b]{2}{*}{ Factor } & & \multicolumn{2}{|c|}{ Provision of dysphagia rehabilitation } & \multirow{3}{*}{$P$-value* } \\
\hline & & Yes & No & \\
\hline \multirow{3}{*}{ Number of patients per month } & & & & \\
\hline & $<35$ & $36(58.1)$ & $26(41.9)$ & \multirow{2}{*}{0.027} \\
\hline & $\geq 35$ & $49(76.6)$ & $15(23.4)$ & \\
\hline \multicolumn{5}{|l|}{ Origin of DDC request } \\
\hline \multirow[t]{2}{*}{ Hospital } & Yes & $46(75.4)$ & $15(24.6)$ & \multirow{2}{*}{0.017} \\
\hline & No & $43(55.8)$ & $34(44.2)$ & \\
\hline \multicolumn{5}{|l|}{ Visiting site } \\
\hline \multirow[t]{2}{*}{ Elder care facility } & Yes & $77(70.6)$ & $32(29.4)$ & \multirow{2}{*}{0.007} \\
\hline & No & $10(41.7)$ & $14(58.3)$ & \\
\hline \multirow[t]{2}{*}{ Hospital } & Yes & $59(73.8)$ & $21(26.2)$ & \multirow{2}{*}{0.013} \\
\hline & No & $28(52.8)$ & $25(47.2)$ & \\
\hline \multicolumn{5}{|c|}{ Cooperation with other professionals } \\
\hline \multirow[t]{2}{*}{ Primary care doctor } & Yes & $74(69.8)$ & $32(30.2)$ & \multirow{2}{*}{0.017} \\
\hline & No & $15(46.9)$ & $17(53.1)$ & \\
\hline \multirow[t]{2}{*}{ Nursing care professional } & Yes & $80(67.8)$ & $38(32.2)$ & \multirow{2}{*}{0.049} \\
\hline & No & $9(45.0)$ & $11(55.0)$ & \\
\hline
\end{tabular}

*Chi-square test.

DDC: domiciliary dental care.

Table 4 Multiple logistic regression analysis using a step-wise method for provision of dysphagia rehabilitation

\begin{tabular}{lccc}
\hline & Odds ratio & $95 \%$ CI & $P$-value \\
\hline Number of patients per month & 2.689 & $1.001-7.223$ & $0.049^{*}$ \\
Origin of DDC request & & & \\
$\quad$ Regional dental association & 0.550 & $0.229-1.318$ & 0.180 \\
$\quad$ Primary care dentist & 0.309 & $0.069-1.387$ & 0.125 \\
$\quad$ Hospital & 2.052 & $0.796-5.290$ & 0.137 \\
$\quad$ Elder care facility & 1.943 & $0.643-5.873$ & 0.239 \\
Visiting site & & & \\
$\quad$ Elder care facility & 1.441 & $0.420-4.939$ & 0.561 \\
Cooperation with other professionals & & & \\
$\quad$ Primary care doctor & 3.295 & $1.117-9.718$ & $0.031^{*}$ \\
$\quad$ Nursing care professional & 1.382 & $0.376-5.078$ & 0.626 \\
Attitude toward accompanying DTs & 1.631 & $0.675-3.943$ & 0.277 \\
\hline
\end{tabular}

CI: confidence interval, DDC: domiciliary dental care.

DTs: dental technicians.

*Statistically significant (i.e., $P<0.05$ ).

hospitals, visiting more elder care facilities and hospitals, and cooperating more with primary care doctors and nursing care professionals. Moreover, multiple logistic regression analysis of provision of dysphagia rehabilitation yielded an odds ratio of 2.689 (95\% confidence interval, 1.001-7.223) for number of patients and 3.295 (1.117-9.718) for cooperation with primary care doctors (Table 4).

\section{Need for oral appliances used in dysphagia rehabilitation}

The rate of respondents with treatment experience using
PAP was $54.3 \%, 92.0 \%$ of whom reported that PAP was effective for improvement of swallowing function (Fig. 4A). However, the number of patients treated was small: $76.0 \%$ of the respondents had treated fewer than 10 patients (Fig. 4B). In contrast, $29.7 \%$ of respondents had treatment experience using PLP, $92.7 \%$ of whom reported that PLP was effective for improvement of oral function. The number of patients treated was even smaller: $75.6 \%$ of the respondents had treated fewer than four patients. 


\section{A. Effectiveness of the oral appliances}

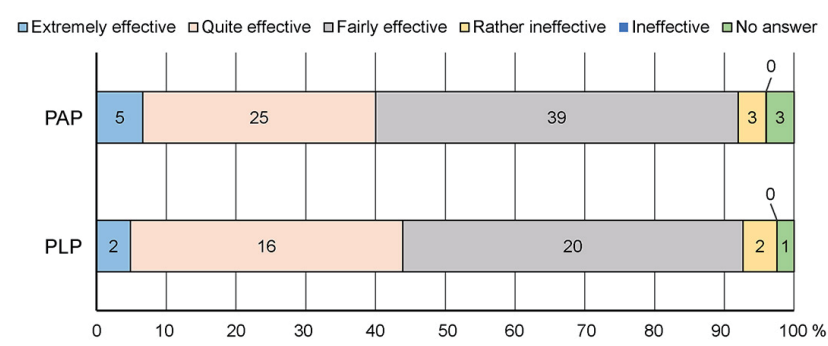

B. Number of cases

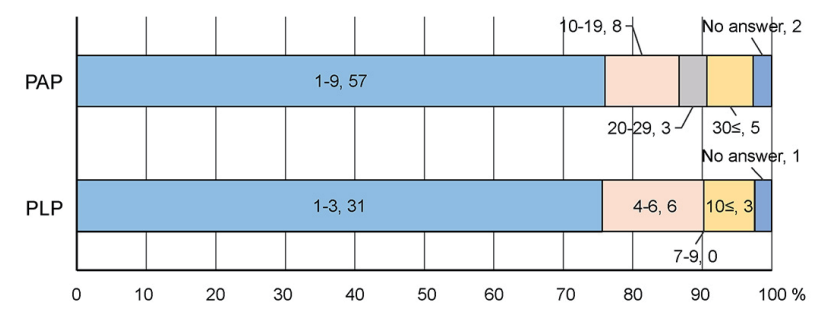

Fig. 4 A: Effectiveness of oral appliances, such as palatal augmentation prosthesis (PAP) and the palatal lift prosthesis (PLP), for oropharyngeal function. The numbers of respondents are indicated in each bar. B: The numbers of PAP and PLP cases are for respondents who performed such treatment. The numbers of cases and respondents are indicated in each bar.

\section{Interprofessional cooperation and team dental care in DDC}

Cooperation with primary care doctors and nursing care professionals in DDC was reported by $76.8 \%$ and $85.5 \%$ of respondents, respectively (Fig. 5A). Regarding team dental practice, only $6.5 \%$ of respondents reported accompanying DTs to DDC, while $92.0 \%$ reported accompanying DHs (Fig. 5B).

Attitudes regarding the need for accompaniment of DTs to DDC were evenly divided: $50.7 \%$ responded positively and $47.8 \%$ negatively. The most frequent reason for a positive response was that collaboration with DTs shortens removable prosthetic treatment time in DDC. Less frequent reasons were that it reduces the dentist's workload and improves prosthesis quality. The most frequent reason for a negative response was the limited need for DT services in DDC.

Respondents were asked about their attitude regarding allowing DTs to touch the inside of patients' mouths, which is currently prohibited by the Dental Technicians Act of Japan: $68.1 \%$ responded positively and $21.0 \%$ responded negatively regarding such permission. A positive attitude to the permission was significantly associated with a positive attitude to accompaniment of DTs to DDC $(P<0.001$, chi-square test).

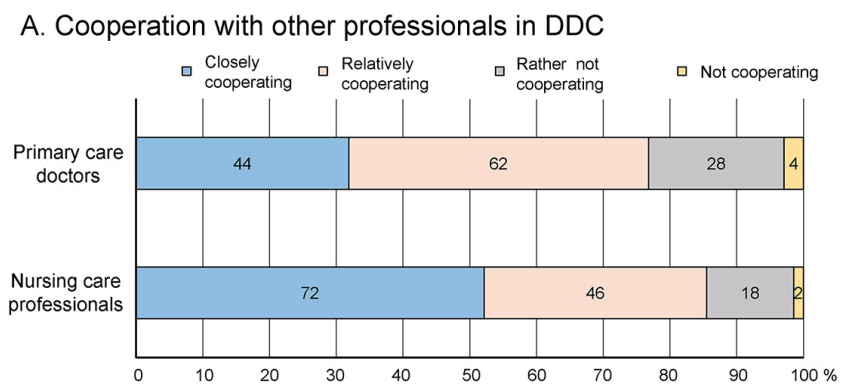

B. Accompaniment of dental professionals

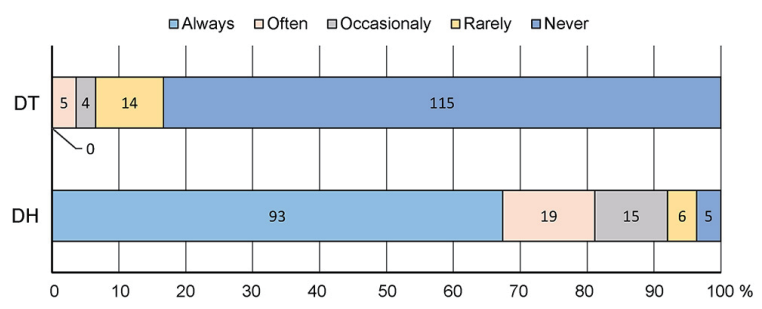

Fig. 5 Interprofessional cooperation and team dental care. A: Cooperation with primary care doctors and nursing care professionals. B: Accompaniment of dental technicians (DTs) and dental hygienists (DHs) to domiciliary dental care (DDC). The numbers of respondents are indicated in each bar.

\section{Discussion}

Previous studies reported relatively low rates of DDC service provision (approximately 30\%) among dental association members in metropolitan areas and thus could not comprehensively describe DDC trends. Therefore, we used a questionnaire survey to investigate DDC services provided by dentists practicing DDC throughout Japan. The respondents reported treating a significantly larger number of patients than those in previous reports, and $27.5 \%$ of the present respondents spent 5 to 7 days per week on DDC suggesting that they were specialized DDC practitioners.

The patient or family, home-visit care station, and hospital were selected as origins of DDC requests by $94.2 \%, 56.5 \%$, and $44.2 \%$ of respondents. These rates are significantly higher than those reported in a previous study (Hirata et al., J Gerodont 27, 276, 2012) (1). With regard to DDC visiting sites, approximately $90 \%$ of respondents reported providing DDC services at a patient's home, which was consistent with the previous report (1). However, the rates of respondents visiting elder care facilities and hospitals were much higher than those in the previous report. These results suggest that cooperation with other healthcare professionals has increased in the 5 years since the previous survey.

The most frequently selected DDC procedure was removable prosthetic treatment, which is consistent 
with previous reports from Japan (1) and Britain (9). The trends of DDC services in the present study were incremental oral care and dysphagia rehabilitation. The increased focus on incremental oral care is likely attributable to heightened recognition that oral care is essential for holistic health and prevention of aspiration pneumonia (2,3,10-14). For this reason, treatment fees for DDC have been revised several times in Japan, and fees for visit dental hygiene instruction provided by an accompanying DH were added to the domiciliary dental service payment schedule (www.mhlw.go.jp/bunya/ iryouhoken/iryouhoken15/d1/h24_01-07-5.pdf). These factors may explain the high percentages of oral care provision and accompanying DHs in the present study. A substantial proportion of respondents, about $65 \%$, reported providing dysphagia rehabilitation. Furthermore, assessment of eating and swallowing function was performed by most respondents $(81.2 \%)$. These results suggest that the respondents were highly motivated to dysphagia rehabilitation because they recognize the importance of improving and maintaining oropharyngeal function in the prevention of aspiration pneumonia and holistic health, as indicated by several previous studies (15-18). Another potential contributing factor is that the 2010 revision of the Japanese health insurance system increased the fee for dysphagia rehabilitation. Besides, functional assessment by VE was performed by $43.8 \%$ of the respondents, which indicates that a considerable number of respondents had acquired competences needed to perform VE and diagnose swallowing disorders.

PAP and PLP are useful for rehabilitation of swallowing and oral function (19-21) and were used by a substantial proportion of the respondents. Virtually all acknowledged the effectiveness of PAP and PLP for improving oropharyngeal function, thereby confirming the benefits of these appliances.

Cooperation with other healthcare professionals is essential in DDC, and a high number of respondents reported cooperating with primary care doctors and nursing care professionals, which indicates that cooperation between various professionals engaged in long-term nursing care has become more common. The importance of team dental care is widely acknowledged, and most respondents reported that they accompanied DHs to DDC. In contrast, most did not accompany DTs, because they felt that DTs had little role in the current DDC paradigm. However, most respondents had positive attitudes about accompaniment of DTs to DDC and future legal permission for DTs to touch the inside of patients' mouths; therefore, there is an expectation that the role of DTs in DDC might expand in the future. To make it realized, DTs should acquire the skills necessary for fabricating appliances such as PAP and PLP, assessing oropharyngeal function, and communicating with patients, their families, and other professionals. They should also increase their knowledge of systemic diseases and their ability to manage difficult cases, which are essential aspects of DDC practice.

Because the present study surveyed dentists who were already providing DDC services, caution is advised when attempting to generalize these results to DDC practices throughout Japan. Nevertheless, the present results likely reflect current trends in DDC, namely, an increase in patient numbers, greater practice of oral care and dysphagia rehabilitation procedures, increased use of oral appliances for dysphagia rehabilitation, and greater cooperation among healthcare professionals engaged in long-term care of patients. These trends are likely to continue because of the rapid aging of the population and progress in integrated community care in Japan.

\section{Acknowledgments}

The authors express their sincere gratitude to the former president of Home Dental Care Net, Dr. Ryuma Hara, and its members for their generous cooperation with this study. We also thank Dr. Masaomi Ikeda, Senior Lecturer, Department of Oral Prosthetic Engineering, Graduate School of Medical and Dental Sciences, Tokyo Medical and Dental University, for his help with statistical analysis, and Ms. Yui Kasai, Graduate, Course for Oral Health Engineering, Faculty of Dentistry, Tokyo Medical and Dental University, for her assistance in collecting data. This study was supported in part by a grant from the Japanese Dental Science Federation (JDSF-DLP-17-2).

\section{Conflict of interest}

The authors have no conflict of interest to declare.

\section{References}

1. Hirata S, Sakayori T, Maki Y, Takano N, Ishii T (2013) Factor analysis on implementation of domiciliary dental care in Metropolitan Tokyo. Bull Tokyo Dent Coll 54, 67-72.

2. Adachi M, Ishihara K, Abe S, Okuda K (2007) Professional oral health care by dental hygienists reduced respiratory infections in elderly persons requiring nursing care. Int J Dent Hyg 5, 69-74.

3. Sakayori T, Maki Y, Hirata S, Okada M, Ishii T (2013) Evaluation of a Japanese "prevention of long-term care" project for the improvement in oral function in the high-risk elderly. Geriatr Gerontol Int 13, 451-457.

4. Baba M, Saitoh E, Okada S (2008) Dysphagia rehabilitation in Japan. Phys Med Rehabil Clin N Am 19, 929-938.

5. Oba S, Tohara H, Nakane A, Tomita M, Minakuchi S, Uematsu $\mathrm{H}$ (2017) Screening tests for predicting the prognosis of oral intake in elderly patients with acute pneumonia. Odontology 
105, 96-102.

6. Tohara H, Saitoh E, Mays KA, Kuhlemeier K, Palmer JB (2003) Three tests for predicting aspiration without videofluorography. Dysphagia 18, 126-134.

7. Wakasugi Y, Tohara H, Hattori F, Motohashi Y, Nakane A, Goto $\mathrm{S}$ et al. (2008) Screening test for silent aspiration at the bedside. Dysphagia 23, 364-370.

8. Osawa A, Maeshima S, Tanahashi N (2013) Water-swallowing test: screening for aspiration in stroke patients. Cerebrovasc Dis 35, 276-281.

9. Sweeney MP, Manton S, Kennedy C, Macpherson LM, Turner S (2007) Provision of domiciliary dental care by Scottish dentists: a national survey. Br Dent J 202, E23.

10. Yoneyama T, Yoshida M, Ohrui T, Mukaiyama H, Okamoto $\mathrm{H}$, Hoshiba $\mathrm{K}$ et al. (2002) Oral care reduces pneumonia in older patients in nursing homes. J Am Geriatr Soc 50, 430-433.

11. Watando A, Ebihara S, Ebihara T, Okazaki T, Takahashi H, Asada M et al. (2004) Daily oral care and cough reflex sensitivity in elderly nursing home patients. Chest $126,1066-$ 1070 .

12. Sarin J, Balasubramaniam R, Corcoran AM, Laudenbach JM, Stoopler ET (2008) Reducing the risk of aspiration pneumonia among elderly patients in long-term care facilities through oral health interventions. J Am Med Dir Assoc 9, 128-135.

13. Sumi Y, Ozawa N, Miura H, Michiwaki Y, Umemura O (2010) Oral care help to maintain nutritional status in frail older people. Arch Gerontol Geriatr 51, 125-128.
14. Maeda K, Akagi J (2014) Oral care may reduce pneumonia in the tube-fed elderly: a preliminary study. Dysphagia 29, 616-621.

15. Sumi Y, Miura H, Nagaya M, Nagaosa S, Umemura O (2009) Relationship between oral function and general condition among Japanese nursing home residents. Arch Gerontol Geriatr 48, 100-105.

16. Sura L, Madhavan A, Carnaby G, Crary MA(2012) Dysphagia in the elderly: management and nutritional considerations. Clin Interv Aging 7, 287-298.

17. Tada A, Miura H (2012) Prevention of aspiration pneumonia (AP) with oral care. Arch Gerontol Geriatr 55, 16-21.

18. Momosaki R, Yasunaga H, Matsui H, Horiguchi H, Fushimi K, Abo M (2015) Effect of dysphagia rehabilitation on oral intake in elderly patients with aspiration pneumonia. Geriatr Gerontol Int 15, 694-699.

19. Okayama H, Tamura F, Kikutani T, Kayanaka H, Katagiri H, Nishiwaki K (2008) Effects of a palatal augmentation prosthesis on lingual function in postoperative patients with oral cancer: coronal section analysis by ultrasonography. Odontology 96, 26-31.

20. Rogus-Pulia N, Rusche N, Hind JA, Zielinski J, Gangnon R, Safdar N et al. (2016) Effects of device-facilitated isometric progressive resistance oropharyngeal therapy on swallowing and health-related outcomes in older adults with dysphagia. J Am Geriatr Soc 64, 417-424.

21. Ohno T, Fujishima I (2017) Palatal and lingual augmentation prosthesis for patients with dysphagia and functional problems: a clinical report. J Prosthet Dent 117, 811-813. 